\title{
The Brushless Doubly Fed Reluctance Machine and the Synchronous Reluctance Machine-A Comparison
}

\author{
Robert E. Betz, Member, IEEE, and Milutin G. Jovanović, Member, IEEE,
}

\begin{abstract}
The brushless doubly fed reluctance machine (BDFRM) is related to the better known brushless doubly fed induction machine (BDFIM). Research into doubly fed machines is motivated by the fact that they allow the use of a partially rated inverter in many variable-speed applications. Research into the BDFRM has been largely ignored in comparison to the BDFIM, despite the fact that it has the potential for greater efficiency as compared to the BDFIM, and the rotor is simpler to manufacture. This paper compares the BDFRM and its singly fed cousin, the synchronous reluctance machine. This is a natural comparison since both machines use the same reluctance rotor. The first part of the paper establishes relationships between the inductances of the two machines. This is then used to facilitate a comparison using the constraints that both machines have the same amount of active material, i.e., the same amount of copper and iron, and that the copper losses for both machines are the same. This analysis also allows an approximate comparison with the conventional squirrel-cage induction machine. The analysis is carried out using machine-independent normalizations.
\end{abstract}

Index Terms-Cascade machines, doubly fed machines, slip recovery, synchronous reluctance machines.

\section{INTRODUCTION}

$\mathbf{T}$ HE brushless doubly fed reluctance machine (BDFRM) is by no means a new machine design. The basic operational concepts were established by Broadway in the early 1970's [1]. Most of this work concentrated on various issues related to the fundamental principles of operation of the machine, some aspects of its construction, and its steady-state performance.

A revival of interest in the BDFRM over the last few years [2]-[8] has been motivated by its slip energy recovery nature which allows an adjustable-speed drive to be constructed with an inverter supplying, at most, half the machine's output power. This could be an important advantage in large drives (in

Paper IPCSD 99-87, presented at the 1999 Industry Applications Society Annual Meeting, Phoenix, AZ, October 3-7, and approved for publication in the IEEE TRANSACTIONS ON INDUSTRY APPLICATIONS by the Electric Machines Committee of the IEEE Industry Applications Society. Manuscript submitted for review June 1, 1999 and released for publication February 2, 2000. The work of R. E. Betz was supported by the Danfoss Visiting Professors Program and Prof. F. Blaabjerg at Aalborg University, Denmark, and the Australian Research Council.

R. E. Betz is with the Department of Electrical and Computer Engineering, University of Newcastle, Callaghan, NSW 2308, Australia (e-mail: reb@ecemail.newcastle.edu.au).

M. G. Jovanović is with the School of Engineering, University of Northumbria at Newcastle, Newcastle upon Tyne NE1 8ST, U.K. (e-mail: milutin.jovanovic@unn.ac.uk)

Publisher Item Identifier S 0093-9994(00)04766-6. particular, with restricted speed range), since the inverter is a major component of the system cost. Furthermore, the lower rating of the power electronics also helps minimize troublesome harmonics injected into the power system. Similar advantages can be obtained using slip energy recovery induction machine drives. The BDFRM offers competitive performance to these systems while having a brushless and cageless rotor. The absence of rotor windings also makes it more efficient and easier to control compared to its brushless doubly fed induction machine (BDFIM) counterpart.

Potential application areas for BDFRM-based drive technology include turbo machinery, variable-speed constant-frequency (VSCF) hydro and wind power applications, commercial and industrial heating, ventilation, and air conditioning (HVAC) applications, and pump applications. The use of BDFRM/IM for wind power generation is being seriously considered since a variable turbine speed system can be designed with a modest cost for the inverter. An additional merit is undoubtedly the possibility of power-factor control, which is particularly useful when turbines are connected to weak grid systems.

The BDFRM has two stator windings with different pole numbers and a salient pole rotor similar to that of a synchronous reluctance machine (SYNCREL). Unlike a conventional machine, however, the number of rotor poles is half the total number of stator poles (i.e., the sum of the stator primary and secondary winding pole pairs) [9]. The rotor provides magnetic coupling between the stator windings and the resultant mutual inductance variation with rotor position causes a change of co-energy and, consequently, torque production. Usually, one winding, known as the primary winding, is connected to the mains supply (with fixed voltage and frequency), whereas the other winding is inverter fed and is called the secondary or control winding. Fig. 1 shows the main structure of a BDFRM-based drive. Depending on the operating mode, the inverter may or may not have full regenerative capabilities.

One nice and interesting feature of the BDFRM is that it can function as a conventional induction machine, a synchronous machine, and a doubly excited induction machine. The fact that it can operate as an induction machine by simply shorting the secondary winding means that it has a fail-safe mode of operation in case of inverter failure. This mode is also used for starting the system. If the secondary winding is fed with dc, then the BDFRM behaves as a synchronous machine, and the associated electronics only handle the winding resistive loss power. In 


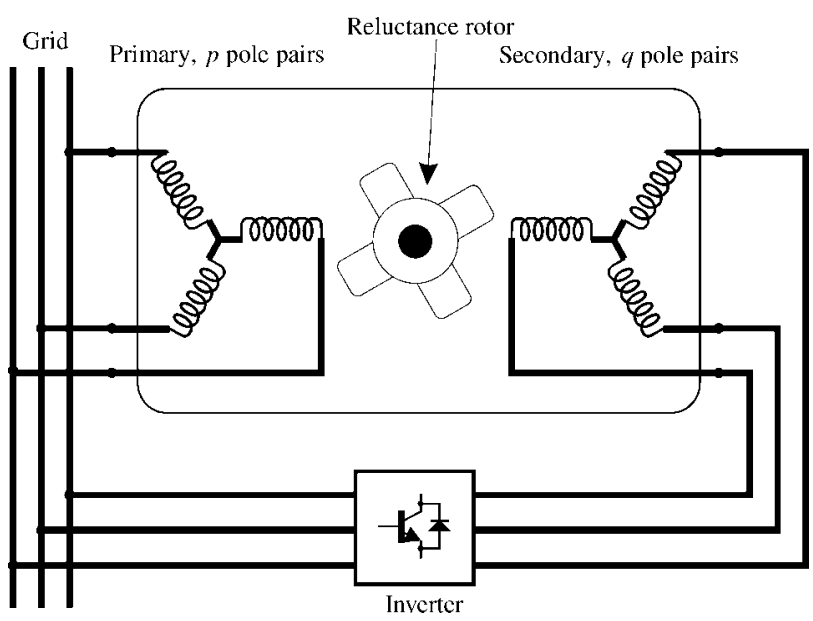

Fig. 1. Block diagram of the BDFRM and its grid and inverter connections.

the situations when the inverter delivers power to the secondary winding, the BDFRM is acting similarly to a doubly fed slip ring induction machine.

Recent work on the BDFRM has focussed on control strategies for the machine [4], [5], [7], [8], [10], and some work has also been done on its design and finite-element modeling [2], [11]. However, a comprehensive theoretical study of the machine properties has not yet appeared. This paper will attempt to make a start on filling this void, although the treatment here is necessarily incomplete due to space limitations and the scope of the comparisons made.

In order to gain a basic understanding of the optimal control properties of this machine, and the tradeoffs between its size and inverter rating, as well as the comparative torque and power of the BDFRM with other machines, we shall compare the BDFRM and its cousin, the SYNCREL. ${ }^{1}$ Where appropriate the comparisons are carried out in a machine independent fashion using normalized analysis techniques (see Appendix B). ${ }^{2}$

Much of the work in this paper requires some background in the space vector theory and $d-q$ modeling of the BDFRM. Good references for this are [3] and [9]. The current angles and frame definitions used in the following discussion appear in Fig. 2.

A few points about the reference frames in relation to the expressions to be presented should be made. The primary $d_{p} q_{p}$ frame is rotating at $\omega$ and the secondary $d_{s} q_{s}$ frame at $\omega_{r}-\omega$ with respect to the stationary $d q$ frame, $\omega_{r}$ representing the angular velocity of the rotor frame (not shown in Fig. 2). By choosing $\omega=\omega_{p}$, the primary frame is obviously rotating at $\omega_{p}$ and the secondary at $\omega_{s}$ (since $\omega_{r}=\omega_{p}-\omega_{s}$ for torque production to exist from the BDFRM [3], [9]), these being the instantaneous primary and secondary applied frequencies. This choice of reference frames greatly simplifies the dynamic equations for this complex machine, allowing the development of understandable steady-state performance expressions.

\footnotetext{
${ }^{1}$ The analysis in this paper implies an ideal machine without saturation and iron losses. It is also assumed that both machines have the same rotor. Note that with the BDFRM it is possible to have odd rotor pole numbers, which cannot occur with the SYNCREL.

${ }^{2}$ Notice that a comparison with the SYNCREL is indirectly a rough comparison with the cage induction machine because of very similar performance of these two machines.
}

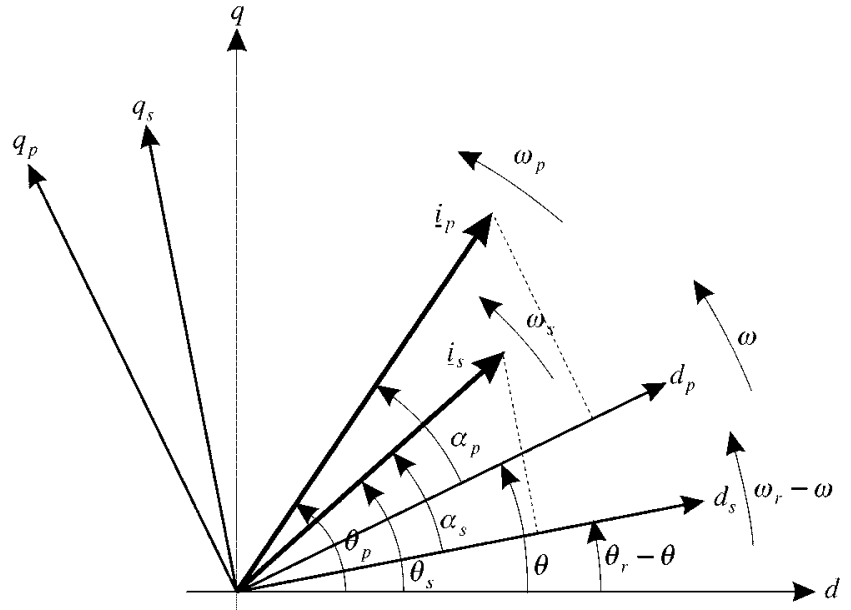

Fig. 2. Reference frames for the BDFRM.

Note 1: The primary reference frame is aligned with the primary flux vector which is constant in magnitude and frequency because the primary is connected to the grid. Therefore, if a current vector is orthogonal to the $d_{p}$ axis, then it does not contribute to flux in the machine.

\section{OPTIMUM PERFORMANCE COMPARISONS}

\section{A. Maximum Torque Per Secondary Ampere (MTPSA)}

A desirable property for a BDFRM may be to optimize the torque produced for a given inverter current rating, i.e., maximize the torque per secondary winding ampere. It can be shown that the electromagnetic torque under the primary flux alignment condition can be expressed as [8], [9]

$$
T_{e_{b m}}=\frac{3}{2} p_{r} \frac{\lambda_{p}}{\zeta} i_{s q}
$$

where $\zeta=L_{p} / L_{p s}$ (refer to Appendix A), ${ }^{3} p_{r} \triangleq$ number of rotor poles, $\lambda_{p} \triangleq$ primary winding flux linkage (a constant), and $i_{s q} \triangleq q$-axis secondary winding current. This equation is very important, as it demonstrates that the machine torque can be controlled via a secondary current component.

From Fig. 2, it can be seen that $i_{s q}=i_{s} \sin \alpha_{s}$ and, hence, the torque per secondary ampere is

$$
\frac{T_{e_{b m}}}{i_{s}}=\frac{3}{2} p_{r} \frac{\lambda_{p}}{\zeta} \sin \alpha_{s}
$$

which is obviously a maximum if the secondary current angle $\alpha_{s}=\pi / 2$.

We can also relate this expression to the rotor design by developing a relationship between the BDFRM inductances and the traditional $d q$ inductances of a SYNCREL. Using (25) and (26), it can be easily shown that the inductances are related as follows:

$$
\begin{aligned}
L_{p} & =\frac{1}{2}\left(L_{d_{p}}+L_{q_{p}}\right) \\
L_{s} & =\frac{1}{2}\left(L_{d_{s}}+L_{q_{s}}\right) \\
L_{p s} & =\frac{1}{2} \sqrt{\left(L_{d_{p}}-L_{q_{p}}\right)\left(L_{d_{s}}-L_{q_{s}}\right)}
\end{aligned}
$$

\footnotetext{
${ }^{3} \mathrm{We}$ desire $\zeta$ to be as small as possible, as this indicates good coupling between the primary and secondary windings.
} 


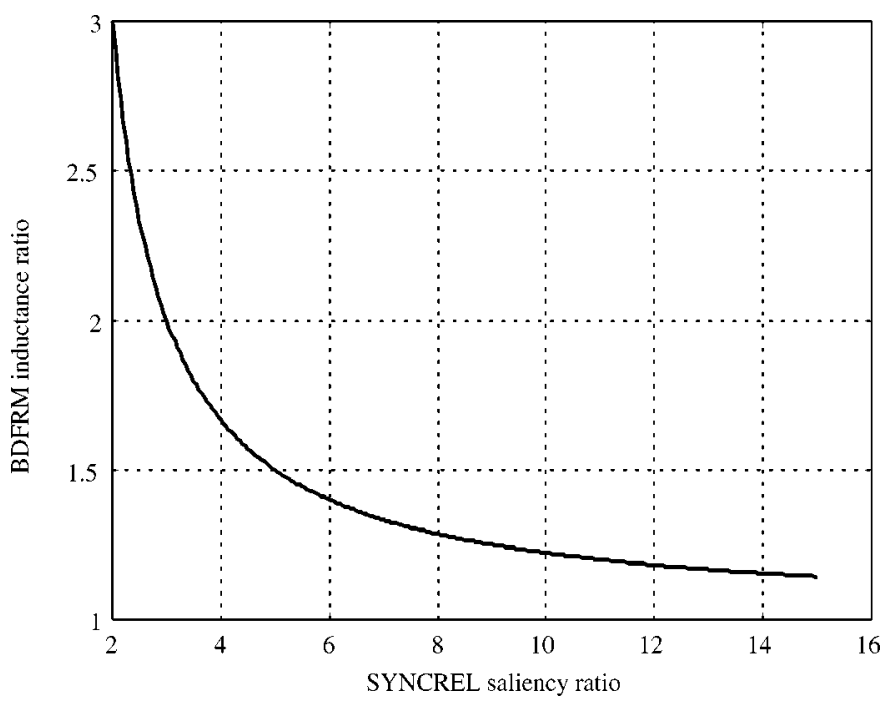

Fig. 3. BDFRM $L_{p} / L_{p s}$ versus SYNCREL $L_{d} / L_{q}$ for $L_{p}=L_{s}$.

where the $p$ and $s$ subsubscripts denote the primary and secondary windings, respectively. ${ }^{4}$ If we assume that the turns/phase/pole for both windings are the same, ${ }^{5}$ then $L_{d_{p}}=L_{d_{s}}=L_{d}$ and $L_{q_{p}}=L_{q_{s}}=L_{q}$ and the MTPSA becomes

$$
\left.\frac{T_{e_{b m}}}{i_{s}}\right|_{\max }=\frac{3}{2} p_{r} \frac{\xi-1}{\xi+1} \lambda_{p}
$$

where $\xi=L_{d} / L_{q}$ is the rotor saliency ratio. Clearly, the MTPSA improves with increased $\xi$ (as do most properties of the SYNCREL) since $\zeta=L_{p} / L_{p s}$ gets smaller (Fig. 3) and, therefore, the primary-secondary magnetic coupling is better. Note that the $(\xi-1) /(\xi+1)$ term above is also the maximum power-factor expression for the ideal SYNCREL.

After considerable manipulation, it is possible to get a torque expression for the BDFRM which bears a close resemblance to that of the SYNCREL [12]

$$
T_{e_{b m}}=\frac{3}{4} p_{r}\left(L_{d}-L_{q}\right) \tilde{z}_{d} i_{s q}
$$

where $\tilde{\imath}_{d}=i_{p d}+(\xi-1 / \xi+1) i_{s d}$. The presence of the second winding produces a larger pseudo $\tilde{\imath}_{d}$ current, resulting in an additional torque in the BDFRM as compared to the SYNCREL.

Forming a ratio of the BDFRM and SYNCREL torques assuming that $i_{p d}=i_{s d}=i_{d}$ and $i_{s q}=i_{q}\left(i_{d, q}\right.$ are the SYNCREL $d q$ currents in the corresponding rotor frame), we obtain the following:

$$
T_{r}=\frac{T_{e_{b m}}}{T_{e_{s y}}}=\frac{2}{1+\frac{1}{\xi}} \geq 1 \quad \forall \xi \geq 1 .
$$

For a typical $\xi=8$, the BDFRM can generate about $78 \%$ more torque than the SYNCREL for the same inverter current and the same equivalent effective turns per pole per phase for all the windings. This, however, is unfair to the SYNCREL since the BDFRM has twice as much copper and copper losses under

\footnotetext{
${ }^{4} L_{d q_{p}}$ and $L_{d q_{s}}$ in the above expressions are defined by (25) for $n_{s y}=n_{p}$ and $n_{s y}=n_{s}$, respectively.

${ }^{5}$ This assumption is equivalent to the BDFRM primary and secondary winding inductances being equal $\left(L_{p}=L_{s}\right)$ as follows from (26).
}

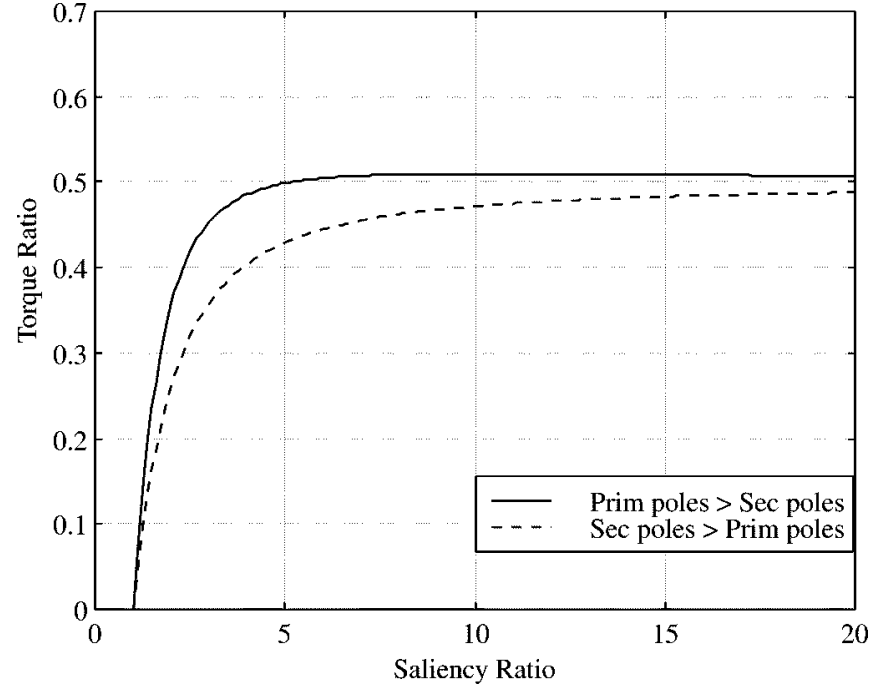

Fig. 4. Torque ratio of a $6 / 2$-pole BDFRM and the 4 pole SYNCREL with the same copper losses, active copper, and a current angle of $\pi / 4$.

this condition. Therefore, the rest of the analysis shall make the following assumption.

Key Assumption 1: The BDFRM has the same gauge wire as the SYNCREL and the same number of total turns, i.e., the sum of the primary and the secondary turns per phase is equal to the turns per phase of the SYNCREL winding. Therefore, the two machines have the same amount of active material. It also implies that the slots are roughly the same size in the two machines and the peak flux densities are approximately the same.

Remark 1: The above assumption is valid if the following phase turns per pole relationship between the machines windings is satisfied [12]:

$$
n_{p}=n_{s}=\frac{n_{s y}}{2} .
$$

Using (9) and setting the primary, secondary, and SYNCREL current angles to $\pi / 4,{ }^{6}$ it is possible to develop the following expression for the torque ratio of the two machines $[12]^{7}$ :

$$
T_{r}=\frac{T_{e_{b m}}}{T_{e_{s y}}}=\frac{1}{2} \frac{\xi^{2}-1}{\xi^{2}+\frac{2 \beta\left(p_{s}-p_{p}\right)}{\beta\left(p_{p}+p_{s}\right)+\pi} \xi+1}
$$

where $\beta \triangleq$ the aspect ratio of the machine, and $p_{p, s} \triangleq$ primary and secondary pole pairs. A plot of this function for a particular pole pair arrangement assuming $\beta=0.5$ (i.e., a NEMA 180 frame) appears in Fig. 4.

Remark 2: If $\xi \rightarrow \infty$ in (10), then $T_{r} \rightarrow 1 / 2$, i.e., the BDFRM is producing half the torque of the SYNCREL.

Remark 3: Note that (10) is slightly strange, in that it has been derived allowing the primary flux to vary (as does the flux in the SYNCREL). The torque ratio is slightly different under the constant flux condition, but, in general terms, the result is the same as above.

\footnotetext{
${ }^{6}$ Note that, in contrast to the SYNCREL, the $\alpha_{p}=\alpha_{s}=\pi / 4$ constraint does not ensure that the BDFRM is producing the maximum torque per ampere (MTPA) in any sense. It is used here primarily because it is the basis for the normalization of the machine.

${ }^{7}$ Note that (9) has to be accounted for in (3)-(5).
} 


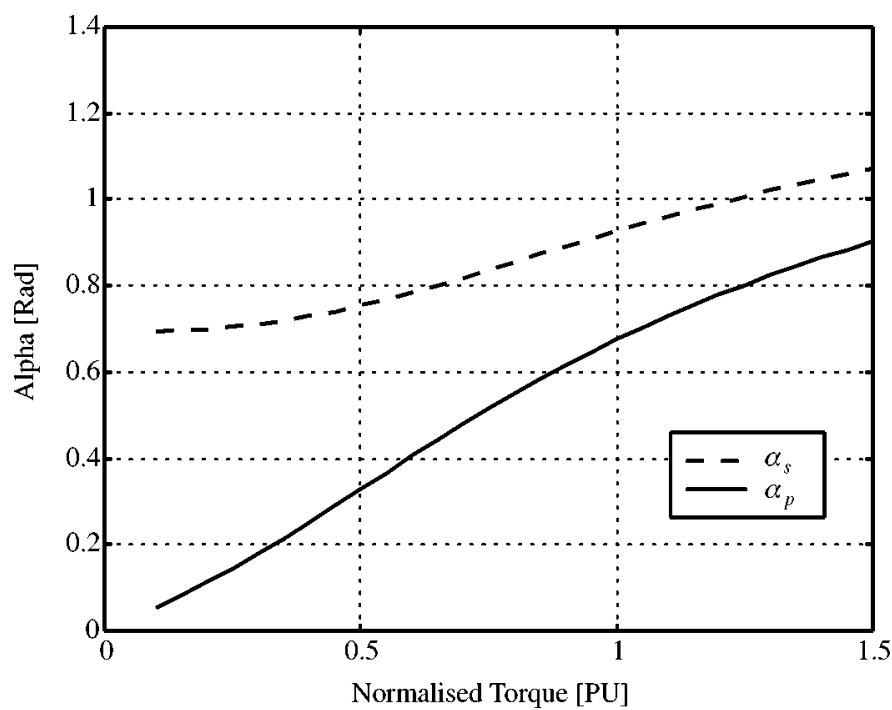

Fig. 5. BDFRM current angles for MTPA.

Remark 4: The observation from Fig. 4 that $p_{p}>p_{s}$ gives a larger $T_{r}$ value does not hold in general. It is only true under the constraint of $\alpha_{s}=\alpha_{p}=\pi / 4$.

\section{B. Maximum Torque Per Total Amperes (MTPTA)}

The previous section considered conditions for MTPSA operation of the BDFRM, but this is not the same as the MTPTA operating point. The latter corresponds to the minimum total current flowing into the machine (i.e., addition of primary and secondary current magnitudes) for a given output torque. It is possible to derive the following expression for the total current input using the normalizations defined in Appendix B [12]:

$$
\begin{aligned}
& i_{t n}=\frac{\zeta T_{n_{b m}} \sqrt{1+\tan ^{2} \alpha_{s}}}{2 \tan \alpha_{s}} \\
&+\frac{\sqrt{\left(2 \tan \alpha_{s}-T_{n_{b m}}\right)^{2}+T_{n_{b m}}^{2} \tan ^{2} \alpha_{s}}}{2 \tan \alpha_{s}}
\end{aligned}
$$

where the secondary current angle $\alpha_{s}$ is a controllable quantity with the normalized torque $T_{n_{b m}}$ being a selected constant value.

A closed-form solution for the optimal $\alpha_{s}$ from the derivative of (11) cannot be obtained and, consequently, numerical techniques have been used. The resultant current angles are shown in Fig. 5.

Remark 5: It is well known that the MTPA for the SYNCREL is achieved at the constant current angle of $\pi / 4$. However, in the case of MTPTA for the BDFRM, the angles are load dependent and change considerably with torque variations.

Remark 6: The $\alpha_{p}$ angle increases significantly with rising torque indicating that the primary current is making an important contribution to the torque production of the machine under this condition. Conversely, because $\alpha_{s}<\pi / 2$, the secondary current is participating more in the flux generation, unlike the MTPSA case where it was purely torque producing (see Note 1 for explanation).

In order to carry out a better comparison with the SYNCREL, we need a normalized SYNCREL torque expression (using the same normalization as the BDFRM). Starting with the standard MTPA expression for the $p_{r}$-pole SYNCREL, we get

$$
T_{e_{s y-\max }}=\frac{3}{8} p_{r}\left(L_{d}-L_{q}\right) i_{s y}^{2} .
$$

Applying (9) to the inductance relationships of (3)-(5), one can deduce that $L_{d}-L_{q}=8 L_{p s}$ and, therefore, (12) can be written as

$$
T_{e_{s y-\max }}=3 p_{r} L_{p s} i_{s y}^{2}
$$

or in the normalized form using the normalizations in Appendix $\mathrm{B}$ as [12]

$$
T_{n_{s y-\max }}=\frac{4}{\zeta} i_{n_{s y}}^{2}
$$

where $i_{n_{s y}} \triangleq$ the SYNCREL current magnitude in pu.

Comparison of different machines is always a difficult process. One means of carrying out a comparison is to assume similar amounts of active material (copper and iron) in the two machines and then compare their output with similar copper losses. We shall attempt this type of comparison here.

It can be established that the relationship between the machine currents for the same copper losses is [12]

$$
\left(\beta p_{p}+\frac{\pi}{2}\right) i_{p n}^{2}+\left(\beta p_{s}+\frac{\pi}{2}\right) i_{s n}^{2}=\left(\beta p_{r}+\pi\right) i_{n_{s y}}^{2}
$$

where $\beta \triangleq$ (length of stack)/(diameter to slot centers of stack). If we assume that $\beta=0.5$ as before, then the above expression can be rearranged as

$$
\begin{aligned}
& i_{n_{s y}}=\sqrt{0.4 i_{p m}^{2}+0.6 i_{s n}^{2}} \quad\left(p_{p}=1, p_{s}=3\right) \\
& i_{n_{s y}}=\sqrt{0.6 i_{p n}^{2}+0.4 i_{s n}^{2}} \quad\left(p_{p}=3, p_{s}=1\right) .
\end{aligned}
$$

If we use (16) and (17) together with (14), one can immediately evaluate the SYNCREL torque for given BDFRM currents such that the copper losses in the SYNCREL are identical to those of the BDFRM.

We can also work out the normalized BDFRM torque using (30) and the MTPTA current angles in Fig. 5. Calculating the BDFRM and SYNCREL torques using these equations with $\zeta=$ $9 / 7$ (which corresponds to a SYNCREL inductance ratio $\xi=8$ according to Fig. 3) one can generate the BDFRM versus SYNCREL optimal torque performance plots presented in Figs. 6 and 7.

Remark 7: Fig. 6 does not show the BDFRM in a favorable light. At best, it produces half the torque of the SYNCREL for the same copper losses (this roughly corresponding to the result in Fig. 4). Note that the torque ratio falls off dramatically for low torques; this is due to the fact that the BDFRM requires significant current to maintain constant primary flux, even when there is little or no torque. The equal copper losses condition results in a significant SYNCREL current and, hence, torque, leading to a small torque ratio at low BDFRM torque values.

Remark 8: The two curves in each of Figs. 6 and 7 result from the relationship between the poles of the windings in the BDFRM. Different copper losses occur in these two cases, giving a different SYNCREL current and torque, as follows from (16) and (17). 


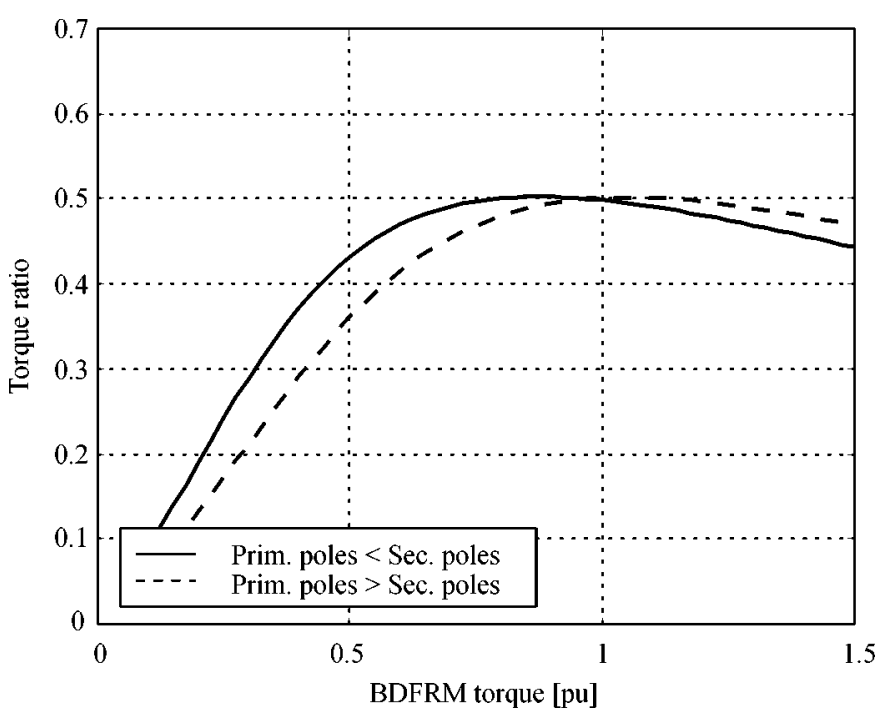

Fig. 6. MTPTA torque ratio between the BDFRM and the SYNCREL with the same copper losses

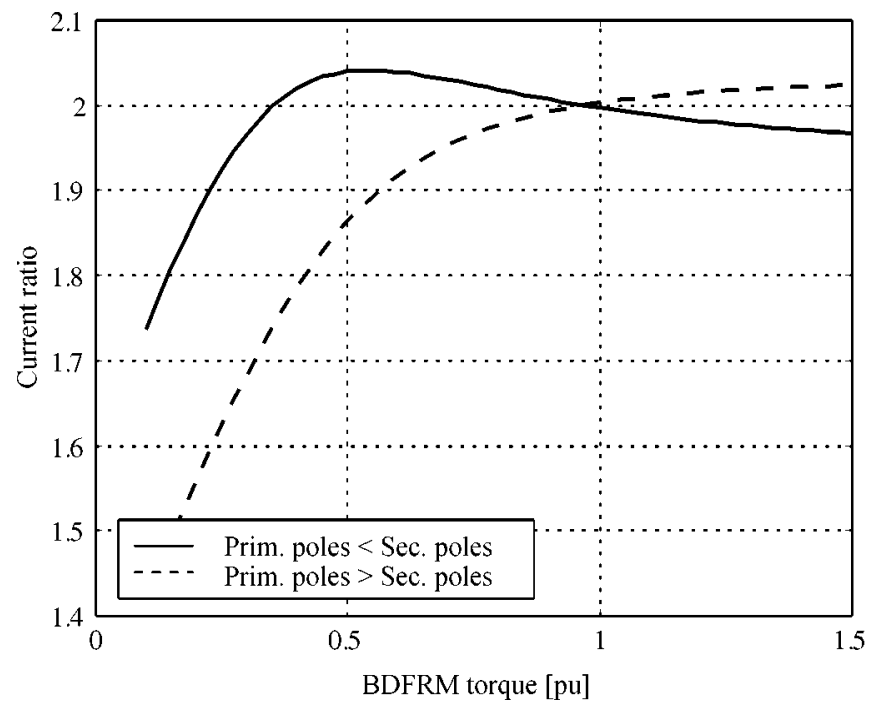

Fig. 7. (BDFRM total current)/(SYNCREL current) versus torque for MTPTA and equal copper losses.

Remark 9: Fig. 7 shows that, for the same copper losses, the BDFRM has twice the total current, while producing half the torque of the SYNCREL. Note that, when $T_{n}<1$ and $p_{p}<p_{s}$, the current ratio is larger. This is because the primary turns/phase $\left(N_{p}=2 p_{p} n_{p}\right)$ and the corresponding winding resistance are then smaller when the majority of the current in the machine is in the primary. Therefore, the copper losses will fall and so will the SYNCREL current (since it is determined based on copper losses) and, consequently, the current ratio will be larger.

Remark 10: Given the lower torque and higher current for the BDFRM, its MTPTA can be shown to be $1 / 4$ that of the SYNCREL under the same copper losses condition.

\section{Power Output and Inverter Rating}

1) Power Output: The previous section was concerned with the comparative torque produced by the machines. However, another equally important aspect of the machine performance

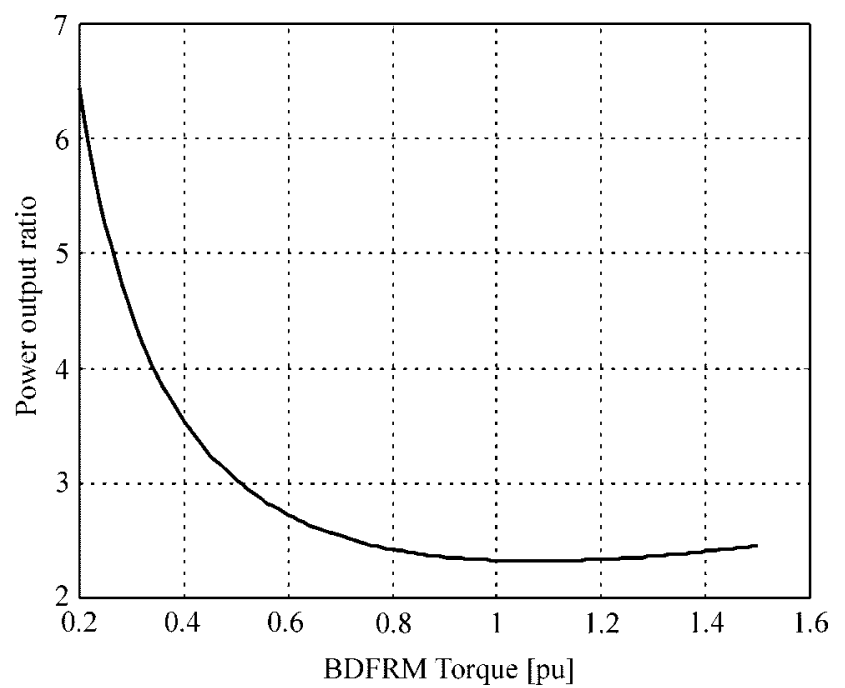

Fig. 8. MTPIA power output ratio of the SYNCREL and the BDFRM for the same copper losses and speed.

is its power producing capability. Key Assumption 1 will again be used. Only the MTPSA control strategy will be discussed, since the secondary current actually determines the inverter rating. ${ }^{8}$ As stated in Section I, a significant motivation for using the BDFRM is the fact that it offers the prospect of a smaller inverter in limited speed range applications. Since the SYNCREL does not have a secondary winding, then we shall call the BDFRM MTPSA strategy the maximum torque per inverter ampere (MTPIA) strategy, so that the term can be applied generically to both machines.

A comparison of the output powers under the conditions of MTPIA is straightforward if the machine shaft angular velocities are equal-it is simply the ratio of the output torques. The relevant power ratio expression for the case when the secondary winding is the multiple pole winding can be shown to be ${ }^{9}$

$$
\frac{P_{\text {out }_{s y}}}{P_{\text {out }_{b m}}}=\frac{T_{n_{s y-\max }}}{T_{n_{b m}}} 4\left[\frac{T_{n_{b m}}^{2}\left(0.1+0.15 \zeta^{2}\right)+0.4}{\zeta T_{n_{b m}}}\right]
$$

which is plotted in Fig. 8. As can be seen from this figure, the best output power of the BDFRM under this condition is about $40 \%$ of that of the SYNCREL. However, this diagram does not give a total evaluation of the performance because it does not account for the voltage available. A better way to compare the machines is to apply the same inverter output voltage to both machines and then determine the corresponding shaft angular velocity and output power. This is a valid approach for comparing performance when both machines use the same size inverter.

Before looking at the same inverter voltage output power in detail, we can observe that the BDFRM windings have half the turns/pole of the SYNCREL windings given (9). Therefore, for the same applied frequency and current, the approximate induced voltage in the BDFRM windings will be about $1 / 4$ that of

\footnotetext{
${ }^{8}$ Remember that, under the MTPSA condition $\alpha_{s}=\pi / 2$, which means that the secondary current only contributes to the torque, and not the flux.

${ }^{9}$ Note that the power ratio is assuming that the SYNCREL is operating in MTPA mode. However, the power output of the SYNCREL can be increased if it is operated in maximum power-factor mode by a factor $\sqrt{2\left(\xi^{2}+1\right)} /(\xi+1)$. For $\xi=8$, this means an increase of approximately $27 \%$.
} 


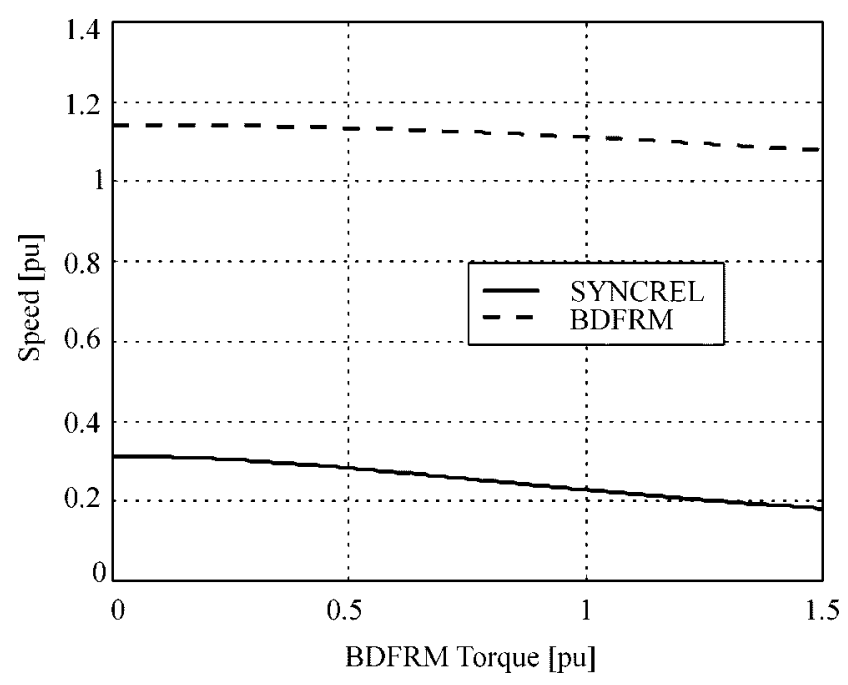

Fig. 9. Rotor angular velocity for the BDFRM and the SYNCREL having the same copper losses and unity per-unit voltage.

the SYNCREL. If the terminal voltages for both machines are the same, then the BDFRM will be able to attain a much higher speed before the inverter runs out of volts. It can be shown that with 1-pu applied voltage and the same copper losses, the normalized shaft angular velocities in terms of the BDFRM torque are [12]

$$
\begin{aligned}
\omega_{r n_{s y}} & =\frac{\zeta}{4 \sqrt{\left(1+\zeta^{2}\right)\left[T_{n_{b m}}^{2}\left(0.1+0.15 \zeta^{2}\right)+0.4\right]}} \\
\omega_{r n_{b m}} & =\frac{1}{2}+\frac{\zeta}{\sqrt{4+T_{n_{b m}}^{2}\left(\zeta^{2}-1\right)^{2}}} .
\end{aligned}
$$

The plots of these functions can be found in Fig. 9.

Remark 11: Note that the BDFRM can rotate at approximately four times the SYNCREL speed before running out of volts. From previous work, we know that it can produce approximately $1 / 2$ the torque of the SYNCREL under the equal copper losses condition. One would, therefore, conclude that it can generate approximately twice the SYNCREL power.

2) Inverter Rating: The inverter size is an important factor in determining the overall cost of any drive system. Therefore, of particular interest is the inverter kilovoltampere rating for the BDFRM and SYNCREL under MTPIA, and with the same copper losses and inverter winding supply voltage.

As in the preceding section, it will be assumed that $v_{n_{s y}}=$ $v_{s n}=1$. Under these conditions, it is possible to show that the normalized kilovoltamperes for the BDFRM and the SYNCREL are:

$$
\begin{aligned}
\mathrm{kVA}_{s y} & =i_{n_{s y}} \\
& =\sqrt{T_{n_{b m}}^{2}\left(0.1+0.15 \zeta^{2}\right)+0.4} \\
\mathrm{kVA}_{b m} & =i_{s n} \\
& =\frac{T_{n_{b m}} \zeta}{2} .
\end{aligned}
$$

Fig. 10 shows the corresponding graphs.

Remark 12: Fig. 10 shows that the inverter current for the BDFRM is substantially lower than that of the SYNCREL under the 1-pu voltage and same copper losses condition.

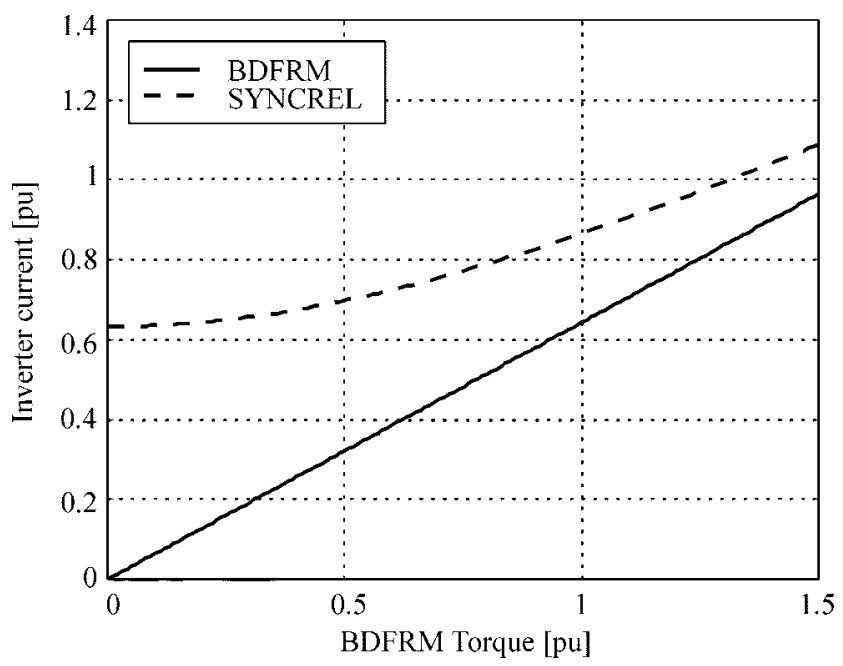

Fig. 10. Optimized inverter-fed winding currents for the BDFRM and the SYNCREL with the same copper losses and 1-pu inverter voltage.

It can be shown using the normalizations defined in Appendix $\mathrm{B}$ that the maximum power is [12]

$$
\begin{aligned}
P_{\max _{s y}} & =\sqrt{\frac{T_{n_{b m}}^{2}\left(0.1+0.15 \zeta^{2}\right)+0.4}{1+\zeta^{2}}} \\
P_{\max _{b m}} & =\frac{T_{n_{b m}}}{2}+\frac{\zeta T_{n_{b m}}}{\sqrt{4+T_{n_{b m}}^{2}\left(\zeta^{2}-1\right)^{2}}} .
\end{aligned}
$$

These equations are plotted in Fig. 11.

Remark 13: The output power of the BDFRM is greater than that of the SYNCREL for the same copper losses for most BDFRM torque levels. If one compares Fig. 11 with Fig. 10, then one can see that the BDFRM has superior output power for less inverter current as compared to the SYNCREL.

Fig. 12 plots the BDFRM kVA versus its power output under the 1-pu voltage and MTPIA control condition used in the previous plots. If this ratio is calculated for the SYNCREL, it is a constant value of 1.63 , which is roughly three times the value for the BDFRM.

Remark 14: Fig. 12 demonstrates further that the power output per inverter kilovoltampere of the BDFRM is clearly superior to the SYNCREL.

\section{CONCLUSIONS}

The following general conclusions can be drawn from the results presented in this paper.

- At best, the torque produced from the BDFRM is half that of the SYNCREL for the same copper losses in both machines. Therefore, for a required torque output, the BDFRM would have to be a larger machine than a SYNCREL.

- Under low torque conditions, the BDFRM is very inefficient compared to the SYNCREL because of the losses resulting from the rather large current flowing into the primary winding (which is preserving constant flux in the machine).

- Under a fixed inverter size assumption, the BDFRM is able to produce approximately twice the output power of the SYNCREL assuming the same copper losses and active 


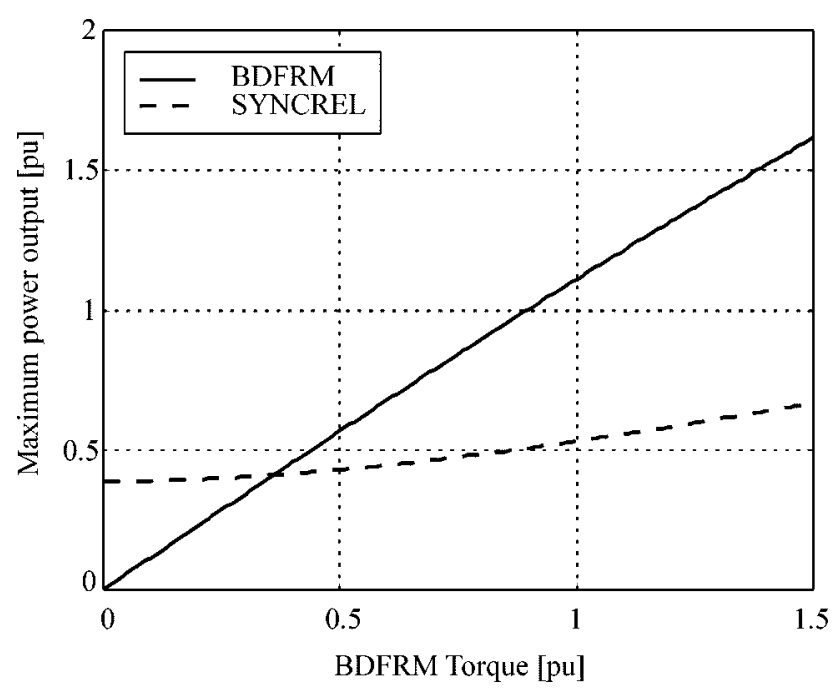

Fig. 11. Output power for a SYNCREL and BDFRM having the same copper losses, 1-pu voltage, and MTPIA control.

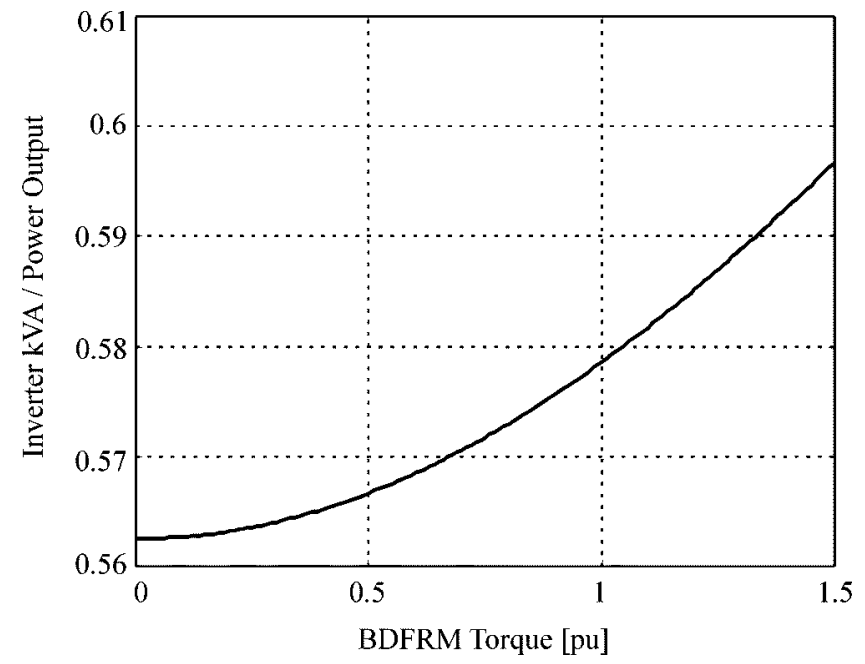

Fig. 12. BDFRM kilovoltamperes versus shaft power ratio with unity per-unit voltage and MTPSA current angle.

copper material, and the control strategy is the MTPIA for both machines. ${ }^{10}$

- In order to achieve a smaller inverter, the BDFRM is controlled in MTPSA mode. This sacrifices some output torque to achieve the reduced inverter rating. The kilovoltampere/(power output) for the BDFRM is approximately $1 / 3$ that of the SYNCREL with the same copper losses.

The preliminary theoretical results presented in the paper indicate that the BDFRM warrants further investigation. It should also be noted that comparisons with the SYNCREL also roughly apply to the induction machine since this machine has similar performance to the SYNCREL. Investigation of machine design and control aspects of the BDFRM is currently in progress, and will be the subject of future papers.

${ }^{10}$ Qualification: The two times factor is only correct when the BDFRM is operating at 1-pu torque and the SYNCREL is operating in MTPIA mode. If the BDFRM torque is less than this, the ratio falls, and if it is greater, then it increases. If the SYNCREL is operating in maximum power-factor mode, the ratio falls, although not dramatically (see footnote 9).

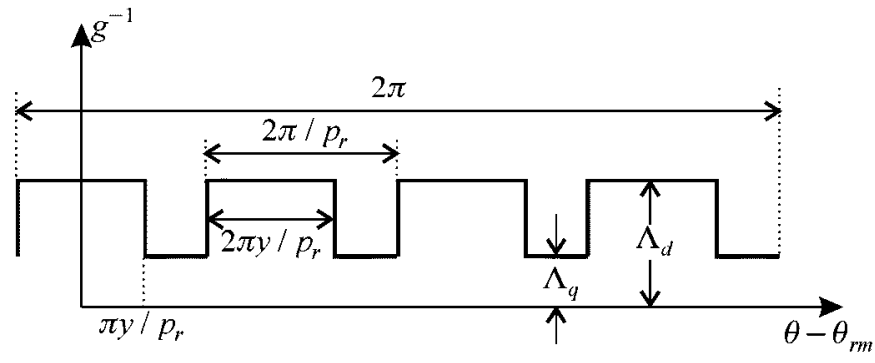

Fig. 13. Inverse air-gap function for a four-pole reluctance rotor $\left(p_{r}=4\right)$ used for inductance determination $(\theta \triangleq$ some mechanical angle around the stator circumference; $\theta_{r m} \triangleq$ the mechanical angle of the rotor high-permeance axis).

\section{APPENDIX A \\ INDUCTANCE RELATIONSHIPS}

The inductance expressions for both the BDFRM and the equivalent SYNCREL listed below have been developed using the method of winding functions [3], [9] with the air-gap parameters as in Fig. 13. It was assumed that both machines had the same rotor and frame size.

\section{A. SYNCREL $d q$ Inductances}

$$
\left.\begin{array}{rl}
L_{d_{s y}} & =\frac{3}{2} \mu_{0} n_{s y}^{2} r l \pi\left(m+\frac{n}{2}\right)+L_{l_{s y}} \\
L_{q_{s y}} & =\frac{3}{2} \mu_{0} n_{s y}^{2} r l \pi\left(m-\frac{n}{2}\right)+L_{l_{s y}} \\
\xi & =\frac{L_{d_{s y}}}{L_{q_{s y}}} \approx \frac{2 m+n}{2 m-n} .
\end{array}\right\}
$$

\section{B. BDFRM Inductances}

$$
\left.\begin{array}{rl}
\text { Primary } L_{p} & =\frac{3}{2} \mu_{0} n_{p}^{2} m \pi r l+L_{l_{p}} \\
\text { secondary } L_{s} & =\frac{3}{2} \mu_{0} n_{s}^{2} m \pi r l+L_{l_{s}} \\
\text { mutual } L_{p s} & =\frac{3}{4} \mu_{0} n_{p} n_{s} n \pi r l \\
\zeta & =\frac{L_{p}}{L_{p s}} \approx \frac{n_{p}}{n_{s}} \frac{2 m}{n} .
\end{array}\right\}
$$

where $n_{s y}, n_{p}, n_{s} \triangleq$ are the effective turns per pole per phase of the SYNCREL and BDFRM windings, respectively; $L_{l_{s y}}, L_{l_{p}}, L_{l_{s}} \triangleq$ are the leakage inductances of the SYNCREL and BDFRM windings, respectively; $r, l \triangleq$ are the machine mean radius and axial length, respectively; $m=y \Lambda_{d}+(1-y) \Lambda_{q} ; n=\frac{2}{\pi}\left(\Lambda_{d}-\Lambda_{q}\right) \sin \pi y ; 1 / \Lambda_{d}$, $1 / \Lambda_{q} \triangleq$ are the minimum and the maximum air-gap lengths, respectively; and $y$ is the pole-arc/pole-pitch ratio of the rotor (Fig. 13). ${ }^{11}$

\footnotetext{
${ }^{11}$ The inductance expressions are valid under the condition that the number of rotor poles is $p_{r}=p_{p}+p_{s}$ and $p_{p} \neq p_{s}$ where $p_{p}$ and $p_{s}$ represent the number of pole pairs of the primary and secondary windings, respectively.
} 


\section{APPENDIX B}

\section{BASE DEFINITIONS AND VALUES}

\section{A. Normalization Bases}

$$
\begin{aligned}
T_{B} & =\frac{3}{4} p_{r} \frac{\lambda_{p}^{2}}{L_{p}} \\
P_{B} & =\frac{2 \omega_{B}}{p_{r} T_{B}} \\
i_{B} & =\frac{\lambda_{B}}{L_{B}}=\frac{\lambda_{p}}{L_{p}} \\
\lambda_{B} & =\frac{v_{B}}{\omega_{B}}=\lambda_{p} \\
\omega_{B} & =\omega_{p}=2 \pi f_{B} \\
L_{B} & =L_{p}
\end{aligned}
$$

where $v_{B}$ and $f_{B}$ are the grid supply voltage and frequency.

\section{B. BDFRM Per Unit Expressions}

Torque

$$
\begin{aligned}
T_{n} & =\frac{2 \sin \alpha_{s} \sin \alpha_{p}}{\sin \left(\alpha_{p}+\alpha_{s}\right)} \\
& =\frac{2}{\zeta} i_{s n} \sin \alpha_{s} \quad \text { (vector control form) }
\end{aligned}
$$

Primary and secondary currents

$$
\begin{aligned}
& i_{p n}=\frac{\sin \alpha_{s}}{\sin \left(\alpha_{p}+\alpha_{s}\right)} \\
& i_{s n}=\frac{\zeta \sin \alpha_{p}}{\sin \left(\alpha_{p}+\alpha_{s}\right)} .
\end{aligned}
$$

Primary and secondary flux linkages

$$
\begin{aligned}
& \underline{\lambda}_{p n}=\underline{i}_{p n}+\frac{1}{\zeta} \underline{i}_{s n}^{*}=\lambda_{p}=1 \\
& \underline{\lambda}_{s n}=L_{s n} \underline{i}_{s n}+\frac{1}{\zeta} \underline{i}_{p n}^{*} .
\end{aligned}
$$

Real power (per winding and total)

$$
\begin{aligned}
P_{p n} & =\frac{1}{\zeta} i_{s n} i_{p n} \sin \left(\alpha_{s}+\alpha_{p}\right)=\frac{1}{2} T_{n} \\
P_{s n} & =\frac{1}{\zeta} \omega_{s n} i_{s n} i_{p n} \sin \left(\alpha_{s}+\alpha_{p}\right)=\frac{\omega_{s n}}{2} T_{n} \\
P_{n} & =P_{p n}+P_{s n}=\frac{1+\omega_{s n}}{2} T_{n}=\omega_{r n} T_{n}
\end{aligned}
$$

where $\zeta=L_{p} / L_{p s}, \omega_{s n}$ is the normalized secondary winding supply frequency, and $\omega_{r n}$ is the normalized rotor angular velocity. ${ }^{12}$

\section{REFERENCES}

[1] A. Broadway, "Cageless induction machines," Proc. Inst. Elect. Eng., vol. 118, pp. 1593-1600, Nov. 1971.

${ }^{12}$ Note that $p_{r} \omega_{r m}=\omega_{p}+\omega_{s}$ where $\omega_{r m}$ is the rotor mechanical angular velocity. It should be also mentioned that $\omega_{p n}=1$ as $\omega_{B}=\omega_{p}$. If $\omega_{s n}=$ 1 , then both BDFRM windings are contributing equally to the machine power output. In this case, obviously, the supply inverter has to handle half the power produced by the machine.

[2] L. Xu, F. Liang, and T. Lipo, "Transient model of a doubly excited reluctance motor," IEEE Trans. Energy Conversion, vol. 6, pp. 126-133, Mar. 1991.

[3] F. Liang, L. Xu, and T. Lipo, "D-q analysis of a variable speed doubly AC excited reluctance motor," Elect. Mach. Power Syst., vol. 19, pp. 125-138, Mar. 1991.

[4] L. Xu and Y. Tang, "A novel wind-power generating system using field orientation controlled doubly-excited brushless reluctance machine," in Conf. Rec. IEEE-IAS Annu. Meeting, 1992, pp. 408-418.

[5] Y. Liao, L. Zhen, and L. Xu, "Design of a doubly-fed reluctance motor for adjustable speed drives," in Conf. Rec. IEEE-IAS Annu. Meeting, vol. 1, Oct. 1994, pp. 305-312.

[6] Y. Liao and C. Sun, "A novel position sensorless control scheme for doubly fed reluctance motor drives," IEEE Trans. Ind. Applicat., vol. 30, pp. 1210-1218, Sept./Oct. 1994.

[7] Y. Tang and L. Xu, "Vector control and fuzzy logic control of doubly fed variable speed drives with DSP implementation," IEEE Trans. Energy Conversion, vol. 10, pp. 661-668, Dec. 1995.

[8] L. Xu, L. Zhen, and E. Kim, "Field-orientation control of a doubly excited brushless reluctance machine," IEEE Trans. Ind. Applicat., vol. 34, pp. 148-155, Jan./Feb. 1998.

[9] R. Betz and M. Jovanović. (1998) "Introduction to brushless doubly fed reluctance machines-The basic equations," Tech. Rep. Dept. Elect. Energy Conversion, Aalborg Univ., Aaalborg East, Denmark. [Online]. Available: http://www.ee.newcastle.edu.au/users/staff/reb

[10] L. Xu and J. Yao, "A compensated vector control scheme of synchronous reluctance motor including saturation and iron losses," IEEE Trans. Ind. Applicat., vol. 28, pp. 1330-1338, Nov./Dec. 1992.

[11] L. Xu, "Analysis of a doubly-excited brushless reluctance machine by finite element method," in Conf. Rec. IEEE-IAS Annu. Meeting, 1992, pp. 171-177.

[12] R. Betz and M. Jovanović. (1998) "Comparison of the brushless doubly fed reluctance machine and the synchronous reluctance machine," Tech. Rep. Dep. Elect. Energy Conversion, Inst. Energy Technology, Aalborg Univ., Aaalborg East, Denmark. [Online]. Available: http://www.ee.newcastle.edu.au/users/staff/reb

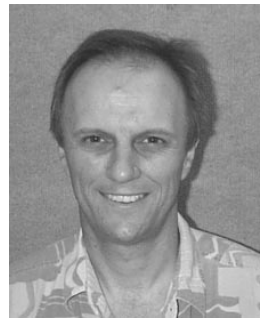

Applications Society.
Robert E. Betz (M'92) received the B.E., M.E., and $\mathrm{Ph} . \mathrm{D}$. degrees from the University of Newcastle, Callaghan, Australia, in 1979, 1982, and 1984, respectively.

He is currently a Senior Lecturer in the Department of Electrical and Computer Engineering, University of Newcastle. His major interests are electrical machine drives, real-time operating systems, and industrial electronics.

Dr. Betz is a member of the Industrial Drives and Electric Machines Committees of the IEEE Industry

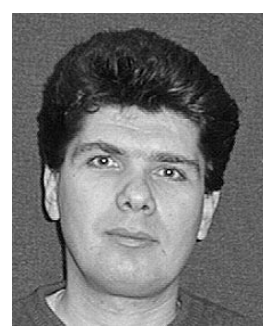

Milutin G. Jovanović (M'99) received the Dipl.Eng and M.E.E. degrees from the University of Belgrade, Belgrade, Yugoslavia, and the Ph.D. degree from the University of Newcastle, Newcastle, Australia, in 1987, 1991, and 1997, respectively.

He is currently a Senior Lecturer in the School of Engineering, University of Northumbria, Newcastle upon Tyne, U.K. His major interests lie in the areas of electrical machines and drives, power electronics, and power systems.

Dr. Jovanović is a member of the Industrial Drives Committee of the IEEE Industry Applications Society. 